\title{
Structuring Interaction in Group Decision Making on Tabletops
}

\author{
Mirko Fetter, Sascha Leicht, David Bimamisa, Tom Gross \\ Human-Computer Interaction Group, University of Bamberg, Germany
}

\begin{abstract}
Group Decision Support Systems allow users to faster reach a consensus and help to improve the overall decision quality by structuring and directing group discussions. We present MTEatsplore, a multitouch tabletop application designed to structure interaction and guide group discussion, and thus improve the decision quality when cooperatively selecting an eating opportunity in a group.
\end{abstract}

\section{Introduction}

Group Decision Support Systems (GDSS) allow users to faster reach a consensus and help to improve the overall decision quality (DeSanctis \& Gallupe 1987) in tasks where a group has to select the best option among several alternatives. GDSS have the potential to structure and direct group discussions, to equalise member participation, to stimulate debate by a meaningful presentation of options, and to generate, organise, and prioritise ideas (Poole et al. 1988). With the advent of horizontal interactive displays like multitouch tabletops and surfaces (e.g. the Samsung SUR40 with Microsoft PixelSense), new means for bringing GDSS to a broader user base arise, providing support for mundane group decisions such as holiday planning. However, in order to adequately support users with their collaborative decision-making tasks, some questions regarding the design space have still to be answered: How to minimise the coordination overhead for turn taking and floor passing? How to support seamless transitioning between solitary and group work? What design principles successfully foster equal participation in the decision-making process? And how to design natural and lightweight mechanisms to support concepts of territoriality and ownership? While some studies (Fetter et al. 2011; Pinelle et al. 2009) have started to shed some light on these issues, the evaluation in these studies is often based on artificial tasks.

In the following we present the multi-touch application MTEatsplore - an interactive, multitouch tabletop application that supports groups of users with selecting among several restaurants an optimal option for the group. It is designed in a way that future studies will allow to get insights in some of the open issues discussed above, based on a real-world task. 


\section{Interaction Concept of MTEatsplore}

MTEatsplore supports a group of users planning to visit a restaurant together with collaboratively making a decision. By structuring their discussion, presenting adequate options and empowering everyone to put forth their position, MTEatsplore supports them with selecting a restaurant that best fits the preferences of all group members. MTEatsplore is optimised for ad-hoc formed groups of 2-4 users and supports various settings as for example in a hotel lobby or at a conference venue. Especially in such ad-hoc formed groups, where the group members do not know each other's preferences, choosing an adequate restaurant can be cumbersome. Particularly when personality traits and attributes like politeness, shyness, or reserve are involved - maybe even with conflicting characteristics of other group members like boldness, forwardness or rashness - finding an optimal decision can become tedious, or even conflict-ridden.
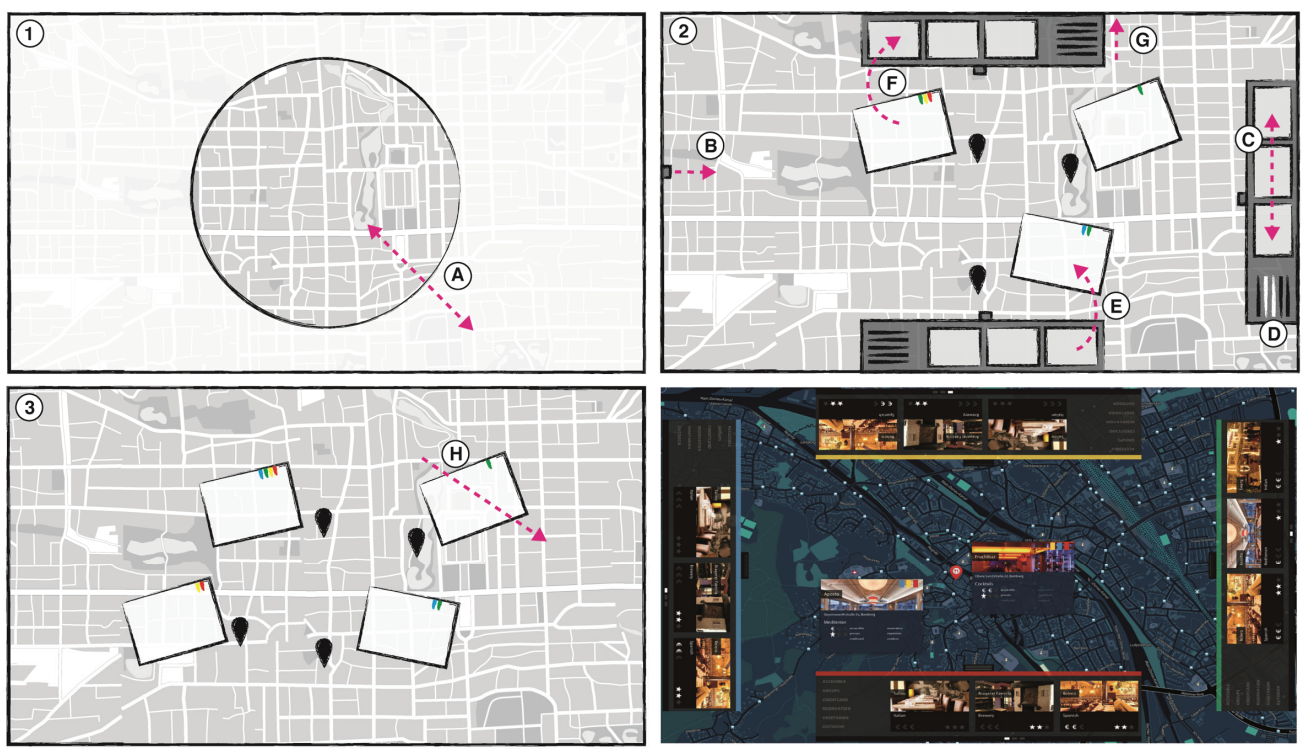

Figure 1. Illustration of the three phases of MTEatsplore, the possible interaction in these phases and a screenshot of the implemented version of the MTEatsplore in the second phase.

Therefore, the interaction concept of MTEatsplore relies on a three-phase model to structure the discussion and equalise participation. The first phase (cf. Figure 1-1) simply allows the group to choose a location and a search radius (A) for the restaurants. This phase is designed for a single user, with the aim is to allow this user-which acts as the informal leader - to take the initiative and thus activate the whole group to use MTEatsplore. When the selection is made, the second phase (cf. Figure 1-2) is automatically started, which now fosters the involvement of all group members. Each of the up to four members can drag out (B) a user workspace at the four sides of the table and then browse through the available restaurants individually by swiping (C). By setting filters (D) the users can reduce the number of restaurants in their workspaces, based on personal preferences (e.g., restaurants with vegetarian options). By dragging (E) a restaurant to the map — which acts as a shared workspace — group 
members can express their interest. When the restaurant sheet is placed on the map, it offers more information and indicates the location. If another user drags out the same restaurant, the two restaurant sheets are visually merged, and a coloured marker at the top of the restaurant sheet indicates which users are interested in this restaurant-each user workspace therefore has assigned one colour (cf. bottom right picture in Figure 1). If users come to the conclusion that they want to reverse their selection they can drag back (F) selected restaurants to their workspaces. This removes their coloured marker from the sheet, and the restaurant reappear in their workspace. In case a user is the only one, who has chosen this restaurant, this action also removes the restaurant sheet from the map. When users are finished with the selection process, they can close $(\mathrm{G})$ their user workspace with a gesture. Once all users closed their user workspaces, the third phase (cf. Figure 1-3) starts. In this phase all selected restaurant sheets are shown on the map, as they were placed in the second phase. The four markers indicate which users are interested in which restaurants, and thus are a good indicator for identifying restaurants the majority of group members prefer. The group now can discuss each restaurant, and conjointly eliminate all options by a strike-through gesture $(\mathrm{H})$ performed by one of the users, until only one restaurant is remaining. As the first phase initiates interaction, and the second phase fosters participation, the last phase uses the momentum of the previous phases, to smoothly guide the users to a final decision.

\section{Design and Implementation of MTEatsplore}

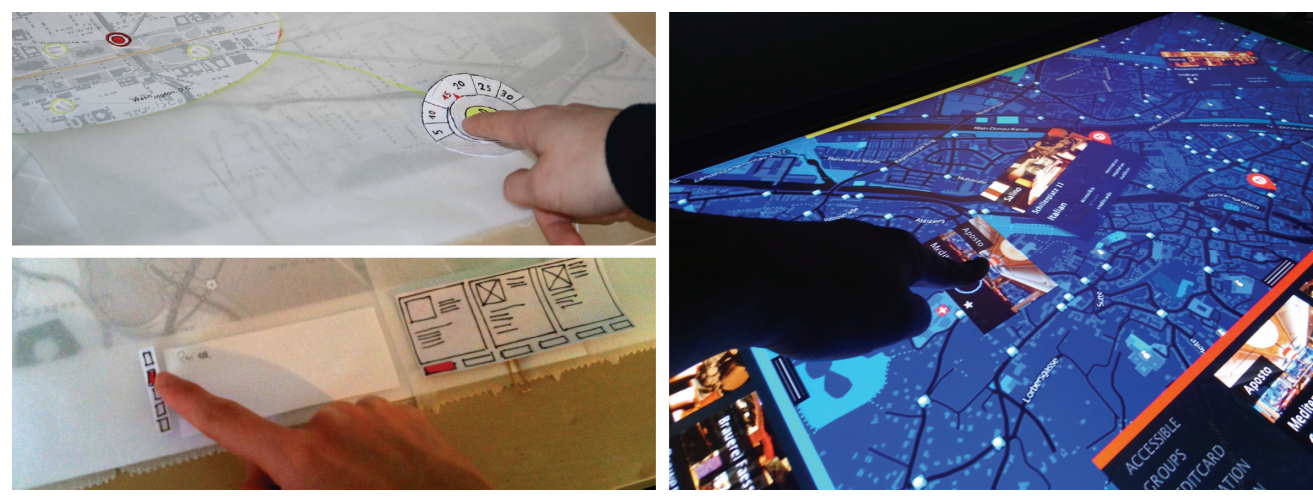

Figure 2. Two photos of the paper prototype (left) and one photo of the final application running on a multi-touch tabletop setup (right).

The interaction concept of MTEatsplore was developed in an iterative design process that involved different prototyping stages from storyboarding, via paper mock-ups (cf. Figure 2) and mid-fi design prototypes to an interactive wireframe prototype and the final implementation (cf. Figure 2). The implementation is done in Java on basis of the MT4j - Multitouch for Java framework (mt4j.org) and supports the TUIO protocol (tuio.org). The application was developed and tested on a setup consisting of an 40" LCD screen that was laid out flat on a regular table in combination with a Microsoft Kinect depth-sensing camera mounted approximately $80 \mathrm{~cm}$ above the screen. Touches were recognised based on the approach suggested by Wilson (Wilson 2010). The LCD screen and the Kinect camera were connected to a 2,7 GHz Quad-Core Intel Core i5 iMac with OS X 10.8.2 and Java 6 installed, that ran the 
MTEatsplore application as well as the touch recognition. As the MTEatsplore application supports the TUIO protocol and is implemented in Java, the application runs on most of the commercial available multi-touch tabletops (like e.g., the Samsung SUR40).

\section{Conclusion and Future Work}

We provided insights in the interaction concept and implementation of MTEatsplore. In future work, studies will analyse parameters like participation, turn taking, or quality of the decision in order to verify if the interaction concepts help to improve group decision making.

\section{References}

DeSanctis, G. and Gallupe, R.B. (May 1987). A Foundation for the Study of Group Decision Support Systems. Management Science 33, 5. pp. 589-609.

Fetter, M., Gross, T. and Hucke, M. (2011). Supporting Social Protocols in Tabletop Interaction through Visual Cues. In Proceedings of the Thirteenth IFIP TC.13 International Conference on Human-Computer Interaction - INTERACT 2011 (Sept. 5-9, Lisbon, Portugal). Springer, Heidelberg. pp. 435-442.

Pinelle, D., Barjawi, M., Nacenta, M. and Mandryk, R. (2009). An Evaluation of Coordination Techniques for Protecting Objects and Territories in Tabletop Groupware. In Proceedings of the Conference on Human Factors in Computing Systems - CHI 2009 (Apr. 4-9, Boston, MA, USA). ACM Press, New York, NY, USA. pp. 2129-2138.

Poole, M.S., Homes, M. and DeSanctis, G. (1988). Conflict Management and Group Secision Support Systems. In Proceedings of the 1988 ACM Conference on Computer-Supported Cooperative Work - CSCW 1988 (Sep. 26-28, Portland, OR, USA). ACM Press, New York, NY, USA. pp. 227-243.

Wilson, A.D. (2010). Using a Depth Camera as a Touch Sensor. In Proceedings of the 2010 ACM International Conference on Interactive Tabletops and Surfaces - ITS '10 (Nov. 7-10, Saarbruecken, Germany). ACM Press, New York, NY, USA. pp. 69-72.

\section{Acknowledgements}

We thank the members of the Cooperative Media Lab.

\section{Contact}

Prof. Dr. Tom Gross, tom.gross(at)uni-bamberg.de, T. 0951/863-3940 